\title{
Light-coupling masks for lensless, sub-wavelength optical lithography
}

\author{
Heinz Schmid, Hans Biebuyck, ${ }^{\text {a) }}$ and Bruno Michel \\ IBM Research Division, Zurich Research Laboratory, 8803 Rüschlikon, Switzerland \\ Olivier J. F. Martin \\ Institute for Field Theory and Microwave Electronics, Swiss Federal Institute of Technology (ETH), \\ 8092 Zurich, Switzerland
}

(Received 1 December 1997; accepted for publication 12 March 1998)

\begin{abstract}
Light-coupling masks (LCMs) based on structured organic polymers that make conformal contact with a substrate can constitute an amplitude mask for light-based lithographies. The LCM is exposed through its backside, from where the light is differentially guided by the structures towards the substrate. Images of arbitrarily shaped features having dimensions much smaller than that of the vacuum wavelength of the exposing light are formed in the resist in a 1:1 correspondence to their size in light-guiding portions of the mask. LCMs allow pattern replication at high resolution and densities over large areas in photoresist without the need for elaborate projection optics. (C) 1998 American Institute of Physics. [S0003-6951(98)04319-8]
\end{abstract}

The principle of coupling and guiding light from one structure into another is based on the idea that materials having matched refractive indices and being in conformal contact allow the propagation of electric fields across their interface without dispersal. Thus by structuring the surface of the contact mask, in-plane discontinuities of the dielectric give rise to localized guiding of light into places where the mask contacts a photoresist. A potential limitation of such lightcoupling masks (LCMs) is the difficulty of achieving uniform coupling between the light-guiding structures and the photoresist. The best coupling requires absolute, or conformal, contact between the two so that no out-of-plane discontinuities result at the interface between the otherwise matched resist and light-guiding structures. This limitation is sufficiently severe that, in general, hard contact lithography with chrome on glass masks has played no significant role in high-resolution lithography despite its potentially attractive simplicity. ${ }^{1}$ The limitation arises from the practical difficulties of placing two hard substrates into conformal contact over large areas without their mechanical breakdown or wear. Rogers et al. ${ }^{2}$ solved this problem by forming the contact mask directly in an elastomeric polymer to obtain "binary phase masks." The method allowed convenient formation of low density features with small $(100 \mathrm{~nm})$ but constant width in an image that reproduced the spatial derivative of the mask. Critical to their application was the height of structures in the mask that defined the phase shifts for this form of interference lithography.

We demonstrate the formation and use of LCMs made from high modulus (10-15 MPa) siloxane polymers ${ }^{3}$ with sufficient strength to define sub-100 $\mathrm{nm}$ features while still allowing their conformal contact with a hard substrate. A structured master is made, typically by electron-beam lithography, that has reliefs in a negative image of the desired pattern. Its replication in the hard siloxane polymer follows by molding the organic directly on this master, with many

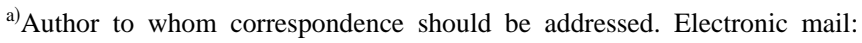
hbi@zurich.ibm.com
}

such replicas possible from a single source. ${ }^{2,4}$ These replicas have sufficient deformability to cause contact with an underlying substrate where gravity and interfacial forces alone create the required intimacy between mask and resist, ensuring good light coupling between them. Their contact is readily reversible and nondestructive because, overall, the structures experience only small forces as the polymers are relatively forgiving and do not propagate stresses as would other brittle, hard materials (e.g., glass). Light originating from a source somewhere above the LCM (in this work) penetrates its bulk and is reflected and guided within the mask towards the underlying photoresist layer, thereby exposing it. Contrast in the exposure between adjoining regions is related directly to the degree of scattering or absorbance at each surface of the structured LCM that is in contact with the substrate. Light that leaks out of the LCM at the air interfaces lowers the contrast of the process and can cause undesired interference effects owing to the phase shift it experiences compared to guided light. ${ }^{2,5}$ These effects can be suppressed by placing absorbing layers ${ }^{6}$ on the noncontacting areas of the LCM. Figure 1 provides a first experimental demonstration of these concepts, proving their validity.

Independent of the material choices, what physical processes describe and limit the guiding and coupling of light using LCMs? We first investigated field distributions in a model light-coupling structure in contact with a simulated resist. Our calculations are based on a Green's tensor technique that solves the three-dimensional integral representation of the photon scattering problem. ${ }^{7}$ This approach takes into account the entire range of evanescent and traveling field components and thereby offers a complete and accurate description of the propagation and scattering of the field at scales both much narrower and much longer than the wavelength. The calculation thus provides a self-consistent description of the behavior of light in a continuum dielectric with embedded scattering centers from the near to the far field. Figure 2(a) shows the evolution of the electric field intensity as it passes through the model LCM and couples into the photoresist. The simulation specifies the origin of the 

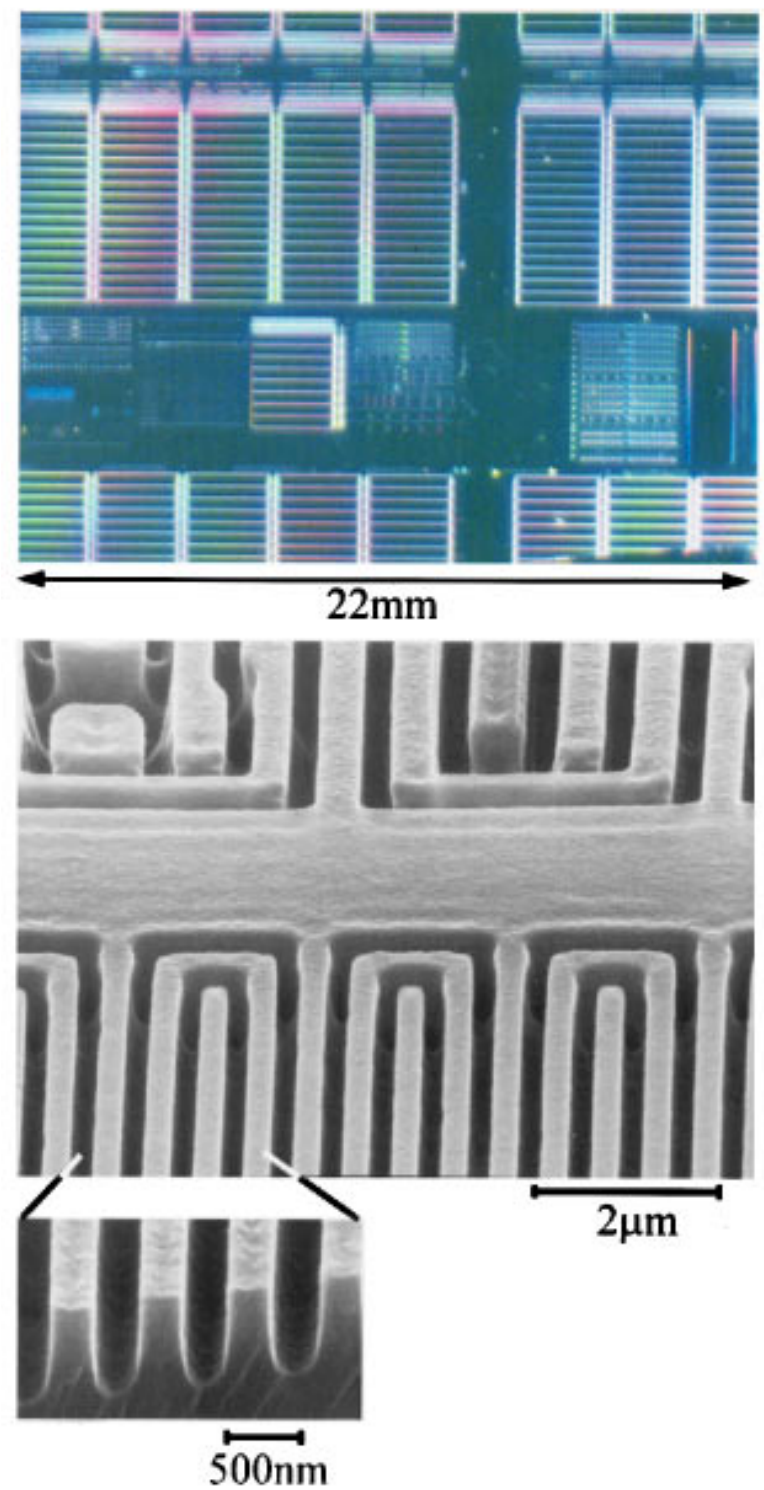

FIG. 1. Use of a light-coupling mask to expose a photoresist at high resolution over a large field. An LCM was placed under its own weight on top of a photoresist (AZ 6612, Hoechst) covered silicon wafer; the resist was exposed through the LCM for $2 \mathrm{~s}$ using $365 \mathrm{~nm}$ light (nominally $5 \mathrm{~mW} / \mathrm{cm}^{2}$ in the plane of the LCM) and formed with the corresponding developer. The upper image shows a partial view of the entire area of exposure $\left(4 \times 4 \mathrm{~cm}^{2}\right.$ in this example), all areas appearing equally well defined in the photoresist. The bottom image, and its inset, show details typical of the resulting structures. Here a thick $(1.4 \mu \mathrm{m})$ resist was used to illustrate the characteristics of the exposure. The LCM was molded from a silicon master with 250-nmdeep reliefs using a siloxane polymer having a modulus of $10 \mathrm{MPa}$; a 5-nmthick gold layer was selectively deposited on the LCM to augment the available contrast.

utility of LCM: conformal contact between the resist and light-guiding structures, along with the air gaps they define, creates regions of strong lateral confinement and amplification of light within the mask. These effects arise because the field prefers to propagate along paths of higher polarizability as it travels through the LCM. The field is therefore effectively focused by the air gaps, concentrated and intensified in the light-guiding part of the LCM, leading to a high contrast in the intensity of light as it emerges into the resist. Importantly, amplification of the field is particularly pronounced at structure sizes of the order of the effective wavelength in the
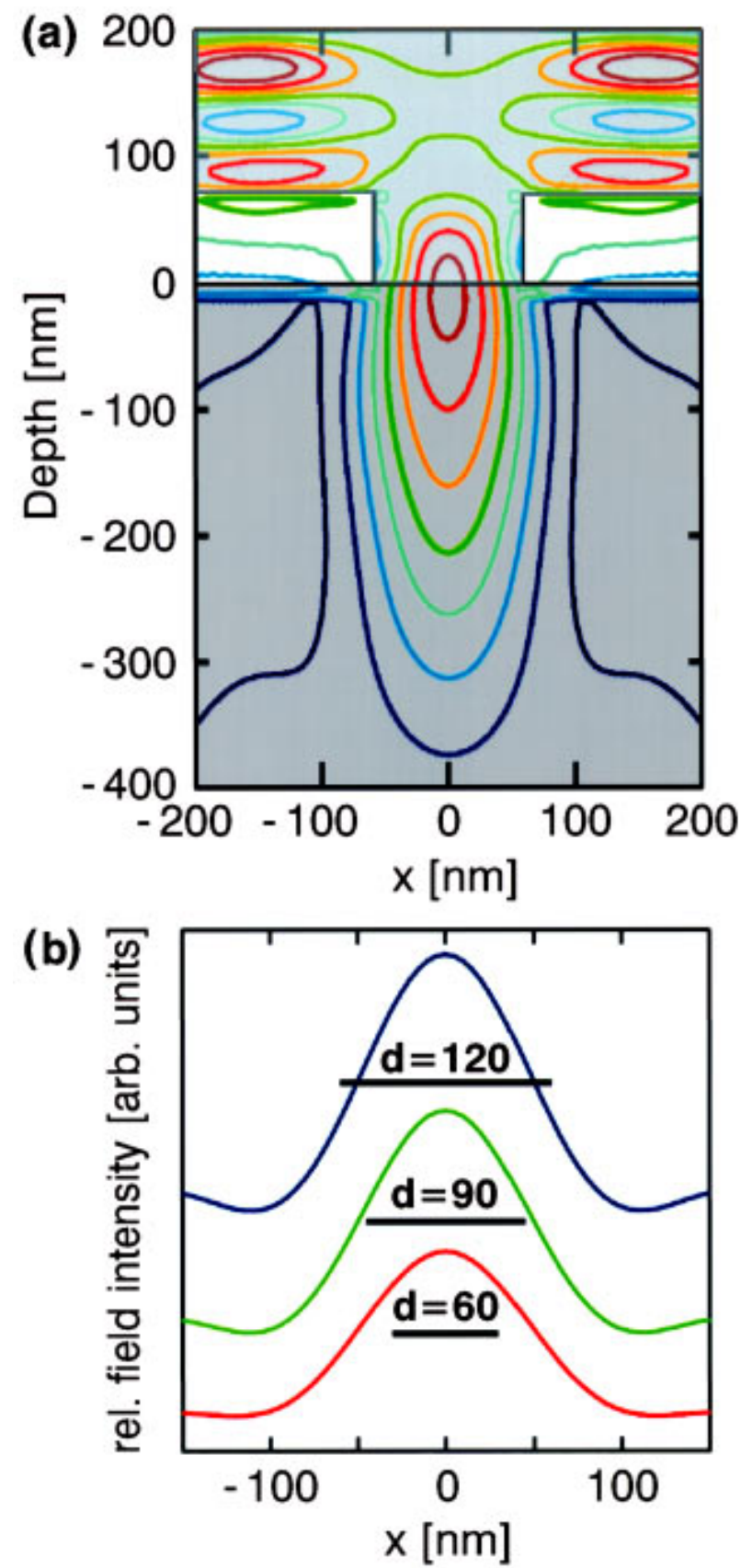

FIG. 2. (a) Simulation (Ref. 9) of the electric field intensity around a 120nm-wide LCM structure contacting a uniform matching dielectric (photoresist). The contours are isointensity lines from a three-dimensonsional (3D) simulation scaled in increments of $20 \%$, where red represents a $60 \%$ increase compared to that of the normalized intensity of incoming light (green). Standing waves in the electric field intensity are evident in the LCM above its air gaps; part of the energy is redirected towards the resist through the contacting area. (b) Simulations like those in (a) were carried out for three light-guiding structures. The relative intensity $25 \mathrm{~nm}$ below the exit aperture of an LCM is plotted for three aperture widths $d$ (shown as horizontal black bars below the curves). A relative increase in intensity of 1.65 , 1.45 , and 1.15 occurs for apertures of 120,90 , and $60 \mathrm{~nm}$, respectively, with a background intensity being 0.2 that of the incoming plane wave.

LCM medium. Structures larger than the wavelength experience progressively less, and in the limit, no such enhancement of light. Such structures can require the use of absorbing or reflecting layers in the nonguiding regions of LCM to achieve differential exposure of the photoresist. Beyond the exit aperture of the LCM, simulations show that the lightguiding structures also act as spatial filters for the field, re- 


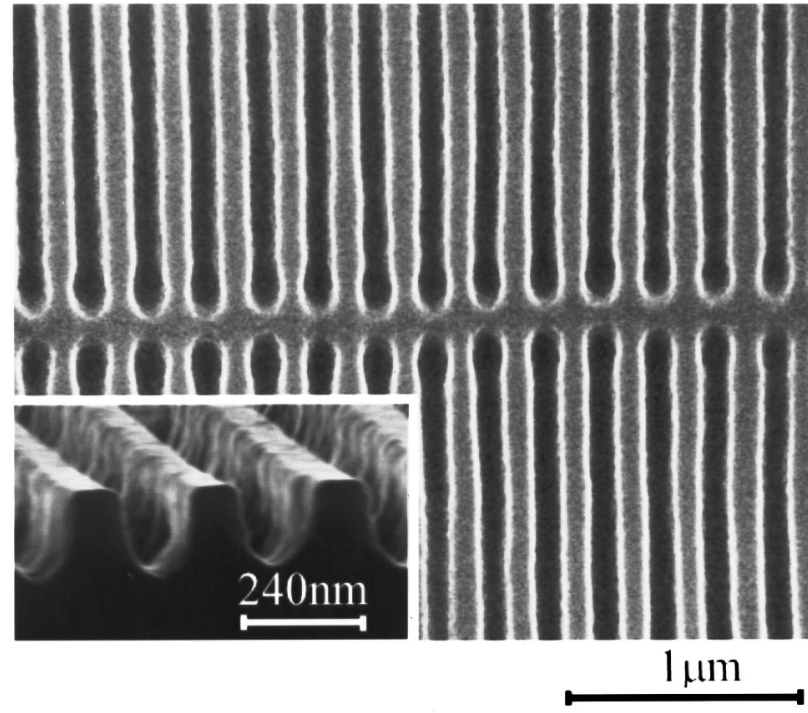

FIG. 3. Example of the resolution and structure sizes readily achievable in photoresist using LCMs at a wavelength in air of $248 \mathrm{~nm}$. The LCM had relief structures $\approx 70 \mathrm{~nm}$ deep and was made with a siloxane-based polymer having a modulus of $15 \mathrm{MPa}$. No additional adsorber (gold) was used in these examples. The field size of exposure was $2 \times 2 \mathrm{~cm}^{2}$.

moving divergent components of light so that it remains focused over a few wavelengths into the resist. Small variations between the index of the resist and that of the LCM do increase the back reflections from their interface but do not substantially effect the degree of lateral confinement of light, substantiating the use of the LCM method in real systems. We found no strong dependence on feature height in achieving light contrast within the mechanical constraints of forming stable features in the LCM. ${ }^{8,9}$

Simulations also reveal that the LCM approach is inherently wavelength-dependent. Figure 2(b) shows that structures substantially smaller than the wavelength in the medium are not readily possible in this implementation of LCMs. Here, wavelength-dependent scattering effects due to dielectric discontinuities become increasingly insufficient to alter the propagation of the plane wave to dimensions corresponding to those of the feature. Thus the image of the lightguiding structure in the photoresist becomes progressively blurred, constraining the resolution limit to half the wavelength in the medium. This result emphasizes the differences between the type of near-field effects dominant in LCMs compared to near-field, evanescent phenomena, the latter remaining highly contained and largely independent of the wavelength but having low intensity. Figure 3 shows an experimental realization of what can be done with LCMs at a wavelength commonly used in optical projection lithography. Particularly striking in these data is the agreement between the description provided by the simulation [Fig. 2(a)] and the photoresist patterns observed (Fig. 3).

Several advantages of the approach discussed and demonstrated above are apparent. The method is simple, requiring no extraordinary apparatus to form patterns in resists at scales down to $100 \mathrm{~nm}$. The effective wavelength of the exposure is that of the light in the medium, smaller than its value in air by the refractive index of the mask. LCMs are useful over a broad range of wavelengths and allow broadband exposures because of the high dielectric contrast of the method, with the resolution limit set by the smallest wavelength. Light contrast through the LCM is boosted particularly for structures at scales of the wavelength, ensuring overall efficiency as the feature size shrinks. No lens is used in the LCM process, eliminating the cost and complexity of the reticles used in conventional optical approaches based on light projection. Moreover, the field size per exposure on the substrate is less constrained by LCMs because aberrations in a system of lenses are no longer an issue. LCMs based on polymers are reusable but are also simple and cheap to replicate from master structures.

Light-coupling masks satisfy the requirements of many single-step lithographic applications (filters, gratings and encoders, for example) and should prove equally useful in mediating other reactions or phenomena requiring light. How these methods will affect the more complicated needs of electronic devices, where alignment of features formed in successive steps is essential, remains open for subsequent investigation. Mask fabrication and its distortion in the replica are clearly concerns requiring further characterization, for example. The simplicity, utility, and economy of the LCM approach nevertheless makes high-resolution, highdensity optical lithography a practical reality in many areas of research and technology.

The authors thank their colleagues A. Beck, A. Bernard, A. Bietsch, E. Delamarche, and H. Rothuizen for their help and discussions. They are particularly indebted to H.R. for his lithography expertise and E. Kiewra for his help with the silicon masters. O.J.F.M. gratefully acknowledges the funding of the Swiss National Science Foundation. The authors also thank P. Guéret (IBM) and R. Vahldieck (ETH Zurich) for their support of the project.

${ }^{1}$ E. Cullmann, Semicond. Int. 8, 332 (1985).

${ }^{2}$ J. A. Rogers, K. E. Paul, R. J. Jackman, and G. M. Whitesides, Appl. Phys. Lett. 70, 2658 (1997)

${ }^{3}$ Not all types of structures can be successfully replicated in elastomeric materials, however [E. Delamarche, H. Schmid, B. Michel, and H. Biebuyck, Adv. Mater. 9, 741 (1997)]. We depended on the formulation of high-strength siloxanes (Young's modulus $>3 \mathrm{MPa}$ ) based on commercially available copolymers (ABCR, Karlsruhe, Germany) V731, HMS301, silane to vinyl terminated ratio $1: 1.5$. 5\% fused silica filler (10-15 nm particle size) was added [B. Michel et al. (unpublished)]. The polymers were translucent down to $230 \mathrm{~nm}$ and absorbed $30 \%$ of the light at $248 \mathrm{~nm}$ for a 3-mm-thick LCM. We found that filled materials substantially more absorbing (97\% of the light) at this wavelength nevertheless formed useful LCMs, underscoring the greatly relaxed optical-material requirements of LCMs compared to traditional (thick) optical elements.

${ }^{4}$ Y. Xia, E. Kim, X.-M. Zhao, J. A. Rogers, M. Prentiss, and G. M. Whitesides, Science 273, 347 (1996).

${ }^{5}$ D. M. Tennant, Microelectron. Eng. 27, 427 (1995).

${ }^{6}$ Thermal evaporation placed a 50 - $\AA$-thick layer of gold on the entire surface of the stamp. Allowing this stamp to contact a gold substrate having a self-assembled monolayer (SAM) of 1,9 nonanedithiol [Aldrich] for $1 \mathrm{~s}$ effected the selective removal of the gold from the surface of the stamp where it contacted the SAM. Such layers were used when features in the mask larger than the vacuum wavelength were targetted (see text).

${ }^{7}$ O. J. F. Martin, C. Girard, and A. Dereux, Phys. Rev. Lett. 74, 526 (1995). ${ }^{8}$ O. J. F. Martin (unpublished results).

${ }^{9}$ The exposing light had a vacuum wavelength of $248 \mathrm{~nm}$ in all of these simulations and a direction of propagation that was normal to the substrate and was circularly polarized in the plane. A mesh size of $10 \mathrm{~nm}$ was employed for the calculation. The resist was treated as having constant optical properties on exposure in these simulations. We assumed an index of 1.6 for the LCM and resist. 